\title{
Different Kinetics Govern Dopaminergic Transmission in the Amygdala, Prefrontal Cortex, and Striatum: An in vivo Voltammetric Study
}

\author{
P. A. Garris and R. M. Wightman
}

Department of Chemistry and Curriculum in Neurobiology, University of North Carolina at Chapel Hill, Chapel Hill, North Carolina 27599-3290

The regulation of extracellular dopamine (DA) concentrations was examined and compared in vivo in four projection fields of mesotelencephalic dopaminergic neurons with fastscan cyclic voltammetry at carbon-fiber microelectrodes. Transient electrical stimulation of ascending DA fibers in a near physiological range of frequencies $(10-20 \mathrm{~Hz})$ elicited similar levels of extracellular DA in the medial prefrontal cortex (MPFC), basal lateral amygdaloid nucleus (BAN), caudate-putamen (CP), and nucleus accumbens (NAC) despite the documented 90-fold disparity in DA tissue levels and terminal density. However, marked differences were observed in the dynamics and overall frequency dependence of the evoked synaptic overflow of DA. These differences are due to the significantly different rates of release and uptake found in each of the four regions. For example, rate constants for the release and uptake of DA were similar in the MPFC and BAN but approximately 8 and 50 times less, respectively, than that in the $C P$ and NAc. When the parameters were normalized to endogenous DA tissue content, a unique picture emerged: compared to all other regions, relative release was 10 -fold greater in the MPFC while relative uptake was at least 10 times less in the BAN. The results further differentiate the functional characteristics of mesotelencephalic dopaminergic systems and demonstrate the regiospecific nature of DA neural transmission in the brain. In addition, the regulation of extracellular DA levels in the MPFC and BAN is suitable for the "long-range" transfer of chemical information in the brain and is consistent with a hypothesis of extrasynaptic neurotransmission.

IKey words: dopamine uptake and release, in vivo voltammetry, amygdala, medial prefrontal cortex, caudate nucleus, nucleus accumbens]

Mesotelencephalic dopaminergic neurons originate in the ventral tegmental area and substantia nigra and converge in the medial forebrain bundle (MFB) before projecting to distinct regions of the telencephalon (Björklund and Lindvall, 1984). This neuronal system is thought to participate in a number of roles, including control of motor activity, motivation, and cog-

Received Mar. 5, 1993; revised June 7, 1993; accepted June 30, 1993.

We gratefully acknowledge M. J. Zigmond, R. W. Keller, and C. D. Kilts for their informative discussions. This research supported by NSF (IBN 9222349) and NIH (NSI 5841).

Correspondence should be addressed to Professor R. Mark Wightman, Department of Chemistry and Curriculum in Neurobiology, CB\# 3290, Venable Hall, University of North Carolina at Chapel Hill, Chapel Hill, NC 27599-3290.

Copyright (C) 1994 Society for Neuroscience $\quad 0270-6474 / 94 / 140442-9 \$ 05.00 / 0$ nition. Dopaminc (DA) has becn implicated in the human neuropathologies of Parkinson's disease and schizophrenia, as well as in the mechanisms underlying drug abuse (Goldman-Rakic, 1984; Vanderwolf et al., 1988; Koob et al., 1989; Carlsson and Carlsson, 1990; Zigmond et al., 1990; Wise and Hoffman, 1992). The various functions of DA are associated with the specific target locations of DA terminal fields (Le Moal and Simon, 1991). In addition, biochemical, pharmacological, and electrophysiological evidence indicates that the subsets of the mesotelencephalic dopaminergic system exhibit very different characteristics (Bannon and Roth, 1983; Glowinski et al., 1984; Kilts et al., 1988).

The regulation of functional levels of DA in mesotelencephalic DA terminal fields has been studied extensively in the rat striatum. In sharp contrast to the rich terminal stores of DA (Anden et al., 1966), extracellular concentrations in situ are in the low nanomolar range (Williams and Millar, 1990b; Ross, 1991; Parsons and Justice, 1992) and are tightly controlled by a delicate balance between release, uptake, diffusion, and degradation (Justice et al., 1988). The relationship of these factors determines, to a great extent, the nature of DA neural transmission. Unfortunately, technical difficulties have precluded a detailed examination of extracellular DA regulation in regions that receive sparse DA innervations and are less accessible to in vivo investigation, for example, using dialysis probes. Thus, our knowledge of the overall regulation of extracellular DA levels and the nature of DA neural transmission in the brain is quite limited.

Transient electrical stimulation of ascending DA fibcrs elicits a rapid change in the concentration of extracellular DA in the striatum (Ewing et al., 1983; Millar et al., 1985; Michael et al., 1987; Suaud-Chagny et al., 1991), which is neurophysiologically relevant (Williams and Millar, 1990a). The technique of fastscan cyclic voltammetry (FSCV) at carbon-fiber microelectrodes can be used to examine these dynamic changes. The evoked responses can be temporally and spatially resolved and, with the aid of a kinetic model, the synaptic overflow curves can be resolved into their respective release and uptake components (Wightman et al., 1988; Wightman and Zimmerman, 1990). With this approach, the extracellular half-life of elcctrically evoked DA in the dorsolateral striatum has been shown to be less than $50 \mathrm{msec}$ due to the rapid removal from the extracellular fluid by the DA uptake system (Wightman and Zimmerman, 1990). Hence, in this region there is little opportunity for the long-range transport of DA from the synapse. This observation is consistent with the suggested role of DA as a classical neu- 
rotransmitter. Comparable information is not available for DA in other areas.

In this study, we employed FSCV to observe the characteristics of DA release and uptake in vivo in projection fields of mesoprefrontal and mesoamygdaloid DA neurons, and contrasted it to that of mesostriatal DA neurons in the rat brain. A more complete characterization of evoked DA overflow in the medial prefrontal cortex (MPFC) (Garris et al., 1993) and basal lateral amygdaloid nucleus (BAN) will be presented elsewhere. Extraction of kinetic parameters from these curves provides the opportunity to compare quantitatively the extent of extracellular DA regulation between regions and to examine the possibility of extracellular transmission by DA.

\section{Materials and Methods}

Animals. Adult, male Sprague-Dawley rats (300-450 gm; Charles Rivers, Wilmington, MA), whose care was in accordance to the Guide for Care and Use of Laboratory Animals (NIH Publication 865-23), were housed under controlled temperature and lighting conditions (12:12 hr light/dark schedule). Food and water were available ad libitum.

Surgery. Rats were anesthetized with urethane $\left(1.5 \mathrm{gm} \mathrm{kg}^{-1}\right)$ and immobilized in a stereotaxic frame (David Kopf Instruments, Tujunga, CA). Temperature was maintained at $37^{\circ} \mathrm{C}$ by isothermal heating pads (Deltaphase Isothermal Pad, Braintree Scientific, Inc., Braintree, MA). Holes were drilled in the skull for reference, stimulating, and working electrodes (Wightman et al., 1988). Two working electrodes were used in all experiments, with one in the striatum and the other in the MPFC or BAN. Implantation employed coordinates obtained from a stereotaxic atlas (Paxinos and Watson, 1986), and are given in millimeters with respect to bregma for the anteroposterior (AP) and mediolateral (ML) locations and from dura for the dorsoventral (DV) positions. The working electrode placements were $+2.7 \mathrm{AP},+0.8 \mathrm{ML},-4.0 \mathrm{DV}$ for $\mathrm{MPFC} ;+1.2 \mathrm{AP},+2.0 \mathrm{ML},-5.0 \mathrm{DV}$ for caudate-putamen (CP) +1.2 $\mathrm{AP},+1.4 \mathrm{ML}, 7.5 \mathrm{DV}$ for nucleus accumbens (NAc); and $2.3 \mathrm{AP}$, $+4.8 \mathrm{ML},-8.3 \mathrm{DV}$ for BAN. The dorsoventral positions given are the average of all placements; in each individual animal the working electrode position was adjusted from these values by no more than \pm 0.5 $\mathrm{mm}$ to observe maximum overflow (May and Wightman, 1989b). The reference electrode rested on dura at approximately +3.0 AP and -4.0 ML.

The bipolar stimulating electrode was normally placed in the MFB $(-4.0 \mathrm{AP},+1.4 \mathrm{ML},-8.0$ to $-9.0 \mathrm{DV})$. In some experiments, the stimulating electrode was placed so that its tips encompassed the ventral tegmental area/substantia nigra region $(-5.3 \mathrm{AP},+0.8 \mathrm{ML},-7.5$ to

$-9.0 \mathrm{DV})$ or the dorsal tegmental bundle $(-5.3 \mathrm{AP},+0.8 \mathrm{ML},-5.0$ to $-6.5 \mathrm{DV}$ ). Placements were histologically verified in some animals. All drugs were administered intraperitoneally.

Electrical stimulation. Electrical stimulation was computcr generated, optically isolated (NL 800, Neurolog, Medical Systems Corp., Great Neck, NY), and applied as biphasic square wave pulses, 2 msec each phase. The stimulation amplitude was normally $300 \mu \mathrm{A}$, which elicits maximal DA release (Wiedemann et al., 1992). An increase in the current amplitude rarely increased the measured response; in animals where an increase did occur the higher amplitude was used for all measurements. A twisted bipolar electrode (Plastics One, Roanoke, VA) with a tip diameter of $0.2 \mathrm{~mm}$ and tip spacing of $1 \mathrm{~mm}$ was employed. The lateral distance of the region stimulated by the electrode is thus approximately $1.4 \mathrm{~mm}$ and was exploited to stimulate DA cell bodies in both the ventral tegmental area and substantia nigra simultaneously. This was verified by the concurrent measurement of evoked DA in both the dorsolateral (i.e., CP) and ventral (i.e., NAc) striatum (data not shown), which receive DA afferents from the substantia nigra and ventral tegmental area, respectively (Gerfen et al., 1987). However, the dorsoventral resolution of the stimulating electrode is much greater (Stamford et al, 1988). For experiments examining the frequency dependence of overflow, the number of stimulation pulses was held constant at 120 unless otherwise noted and the order of the frequencies employed was randomly chosen.

Electrochemistry. Two working electrodes, each implanted in a different dopamine-containing region, were used in each rat. Working electrodes were prepared from carbon fibers $(r=5 \mu \mathrm{m}$; Thornell P-55, Amoco, Greenville, SC), sealed in a pulled glass capillary and polished at $20^{\circ}$ (BV-10 Micropipette Beveler, Sutter Instrument Co., Novato, CA) to yield an elliptical tip. The electrodes were soaked in 2-propanol and dip-coated in $2.5 \%$ Nafion, an ion-exchange polymer that excludes anions such as dihydroxyphenylacetic acid and ascorbate (Baur et al., 1988). The reference electrode was a sodium-saturated calomel electrode (SSCE). Cyclic voltammograms were recorded every $100 \mathrm{msec}$ at a scan rate of $300 \mathrm{~V} \mathrm{sec}^{-1}(-0.4$ to $1.0 \mathrm{~V}$ ) using an El-400 potentiostat (Ensman Instrumentation, Bloomington, IN) under computer control (Wiedemann et al., 1991). The time-dependent current was monitored over the peak oxidation potential for DA in successive voltammograms and converted to concentration based on postcalibration of the electrode in vitro with DA. When necessary, replicate stimulations were averaged to improve the signal-to-noise ratio. For example, detection of $100 \mathrm{~nm}$ changes in DA concentration typically requires averaging four stimulations to achieve a signal-to-noise ratio of 5 . Locally written software synchronized voltammetry and stimulus pulses.

Data analysis. Where applicable, all measurements are reported as the mean \pm SEM. Significance testing used one- and two-way analysis of variance; comparisons between mean values were made by the method of Tukey-Kramer (Sokal and Rohlf, 1981). The significance level was set at $P<0.05$.

Measured curves for the evoked synaptic overflow of DA contain information concerning the release of DA and its uptake (Wightman et al., 1988; Wightman and Zimmerman, 1990). There is also a delay that is evidenced in the time-dependent traces by the continued rise in extracellular DA concentration after the stimulus. In select areas of the CP, this has been shown to be due to diffusion of DA through the Nafion film coating the carbon surface (Kawagoe et al., 1992). To account for this, the permeation through the Nafion film for cach clcctrode was determined as the half-rise time to a concentration pulse after in vivo use. This value, typically $0.6 \mathrm{sec}$, can be used to deconvolve the Nafioninduced delay from the in vivo measurements. The working electrode was positioned in each region to obtain minimum delay times.

Following removal of the Nafion-induced delay, the overflow curves can be described by a simple model (Wightman et al., 1988; Wightman and Zimmerman, 1990): the rising portion of overflow curves (i.e., the appearance of extracellular DA) is described by the rate of release minus the rate of uptake, and the falling portion of overflow curves (i.e., the clearance of extracellular DA) is described by the rate of uptake. Uptake was characterized from the poststimulation clearance of extracellular DA concentrations. In the CP and NAc, uptake was assumed to follow Michaelis-Menten kinetics:

$$
-\{d[\mathrm{DA}] / d t\}_{\text {uplake }}=V_{\text {max }} /\left(K_{\mathrm{m}} /[\mathrm{DA}]+1\right)
$$

A value of $0.16 \mu \mathrm{M}$ was used for $K_{\mathrm{m}}$ (Near et al., 1988; Marshall et al., $1990)$ and $V_{\max }$ was determined from clearance curves where [DA] $\gg$ $K_{\mathrm{m}}$. In this article the rate constant for uptake is given as $k=V_{\max } / K_{\mathrm{ni}}$, the rate constant that is appropriate at the low concentration of $\mathrm{DA}$ normally present in the extracellular fluid of the striatum (Williams and Millar, 1990b; Ross, 1991; Parsons and Justice, 1992).

Uptakc was analyzed differently for the MPFC and BAN because values for $K_{\mathrm{m}}$ are not currently available in the MPFC and BAN of the rat and $K_{\mathrm{m}}$ may not be homogeneous for all DA neurons (Demarest and Moore, 1979; Annunziato et al., 1980; C. D. Kilts, personal communications). Therefore, clearance curves were fit to a first-order process characterized by a rate constant $k$.

$$
-\{d[\mathrm{DA}] / d t\}_{\text {uptake }}=k[\mathrm{DA}]
$$

The majority of data in the MPFC and BAN were $\leq 0.5 \mu \mathrm{M}$ and overflow curves collected in these regions were relatively frequency independent. Thus, this is a rough but reasonable approximation and is supported by the goodness of fit between data and simulations (see below), and the similarities in simulated overflow curves using Michaelis-Menten and first-order uptake using representative kinetic constants for these two rcgions (data not shown).

The rate of release was described as $[\mathrm{DA}]_{n} f$, where $[\mathrm{DA}]_{l}$, reflects the concentration of DA released per stimulus pulse and $f$ is the stimulation frequency. Thus, assuming uptake is an ongoing process during stimulation, the rate of evoked DA overflow is

$$
\{d[\mathrm{DA}] / d t\}_{\text {overtlow }}=[\mathrm{DA}]_{n} f-\{d[\mathrm{DA}] / d t\}_{\text {uplake }}
$$

For each animal, simulations of the measured curves at each frequency were generated by numerical integration of Equation 3 with initial estimates of $[\mathrm{DA}]_{l,}$ and the uptake constants obtained from Equation 1 or 2. The simulations were convoluted with the response time of the 


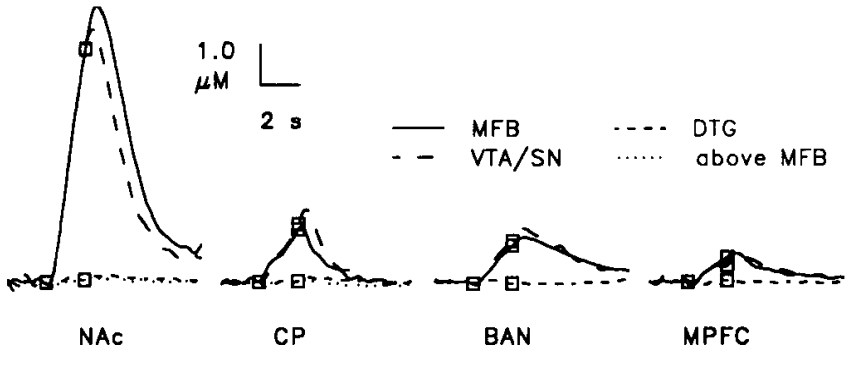

Figure 1. Effects of selective activation of ascending catecholaminergic innervations. Stimulating electrodes were placed in the MFB or the ventral tegmental area/substantia nigra regions ( $V T A / S N$; see Materials and Methods for details). Individual overflow curves are shown for a $60 \mathrm{~Hz}, 2 \mathrm{sec}$ stimulation beginning at time 0 . Initiation and termination of the stimulus are indicated by the open boxes. Data for the CP or NAc during MFB and VTA/SN stimulation were collected with the same working electrode in one rat using two stimulating electrodes. Data for the MPFC or BAN were from two rats. The results of stimulating ascending norepinephrine fibers traversing the dorsal tegmental bundle $(D T G)$ and $0.3 \mathrm{~mm}$ above the DA fibers in the MFB, as evidenced by evoked DA overflow in the CP and NAc, are also shown.

Nafion film (Kawagoe et al., 1992), and compared to the experimental data. The value of [DA], was adjusted for each curve until a best fit was obtained as evaluated by the correlation coefficient.

Reagents. All chemicals were obtained from Sigma Chemical Co. (St. Louis, MO), and were reagent grade and used as received. Solutions were prepared in doubly distilled, deionized water (Mega Pure System, Corning Glasswork, Corning, NY). Drugs were dissolved in physiological saline.

\section{Results}

Stimulation of the $M F B$ evokes release in four areas. Electrical stimulation of the MFB has previously been shown to cause simultaneous release of DA in the CP and NAc (Kuhr et al.,

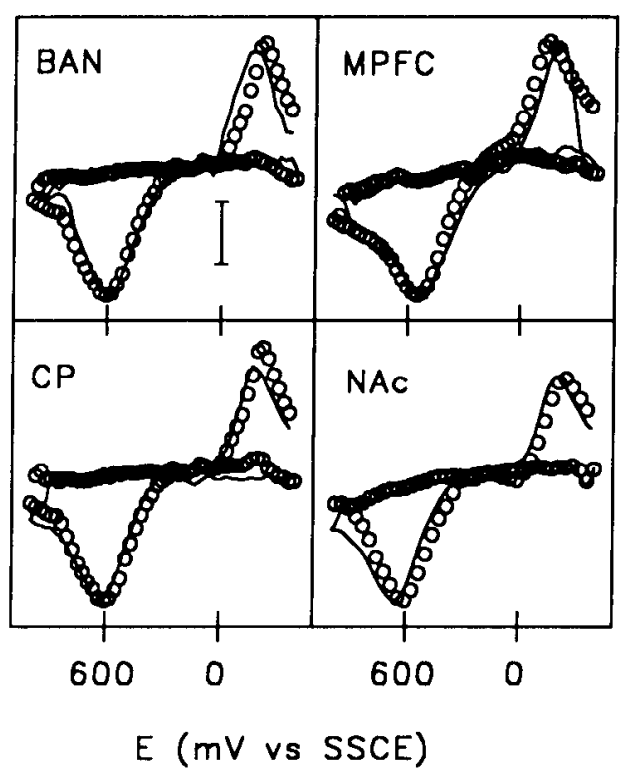

Figure 2. Background subtracted cyclic voltammograms collected in vivo during stimulation and in vitro from DA. Voltammograms from each region (solid lines) are compared to DA collected at the same electrode during postcalibration (open circles) immediately after the experiment. Voltammograms for DA in vitro were normalized with respect to peak oxidation current displayed as negative (i.e., downward) current. Calibration for cyclic voltammograms in vivo is $0.06 \mathrm{nA}$ for $M P F C, 0.08 \mathrm{nA}$ for $B A N, 0.14 \mathrm{nA}$ for $C P$, and $0.14 \mathrm{nA}$ for $N A C$.
CP
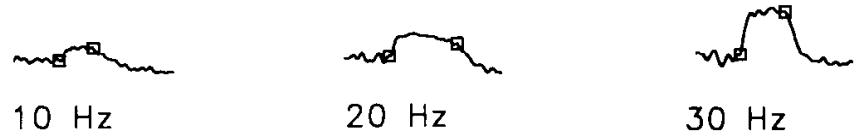

$20 \mathrm{~Hz}$

$30 \mathrm{~Hz}$

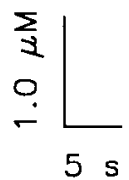

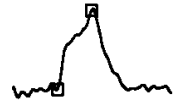

$40 \mathrm{~Hz}$

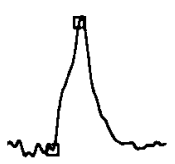

$50 \mathrm{~Hz}$

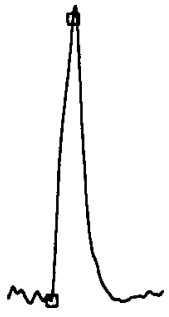

$60 \mathrm{~Hz}$
Figure 3. Frequency dependence of evoked DA overflow in the CP. Individual overflow curves for $10-60 \mathrm{~Hz}$ are shown. The number of stimulus pulses for $10 \mathrm{~Hz}$ was 30 . Open boxes denote initiation and termination of the stimulus.

1986; Stamford et al., 1988). In this work, the position of the stimulating electrode was adjusted to cause maximal DA release with a working electrode in one of the striatal regions, and a second working electrode was then lowered to the MPFC or BAN. During stimulation, synaptic overflow was detected in all areas as a transient increase in the voltammetric current at the potential for DA oxidation that returned to the baseline level after the stimulation (Fig. 1). The response is anatomically specific since no response was obtained in any region when the position of the stimulating electrode was $0.3 \mathrm{~mm}$ dorsal to the MFB.

Identification of the species monitored voltammetrically employed electrochemical, pharmacological, and anatomical criteria (Wightman et al., 1987; Marsden et al., 1988). First, cyclic voltammograms collected in all regions during stimulation were identical with DA and are indicative of a catecholamine (Fig. 2). Thus, ascorbate, dihydroxyphenylacetate (and other DA metabolites), and 5-hydroxytryptamine do not contribute to the measured signal (Baur et al., 1988). In particular, ascorbate and dihydroxyphenylacetate are discriminated against because of the cation exchange polymer on the electrode surface. In addition, extracellular concentrations of these electroactive species do not change with MFB stimulation during the time course of our measurements (Kuhr et al., 1984; Michael et al., 1985). Second, the responses in each region were completely eliminated with the catecholamine synthesis inhibitor $\alpha$-methyl-p-tyrosine (200 $\mathrm{mg} / \mathrm{kg}$; Garris et al., 1993; P. A. Garris and R. M. Wightman, unpublished observations). Ihird, to distinguish between DA and norepinephrine, which are present in similar amounts in the MPFC and BAN (Palkovits ct al., 1979; Kilts and Anderson, 1987), the stimulating electrode was placed in the ventral tegmental area/substantia nigra region (Fig. 1), the location of DA cell bodies. The stimulated response was identical in all regions with that obtained with MFB stimulation. Evoked catecholamine responses did not occur in areas of the MPFC and amygdala, which are relatively norepinephrine rich and DA poor with MFB stimulation (Garris et al., 1993; Garris and Wightman, unpublished observations). Surprisingly, stimulation of the as- 
NAC

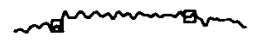

$10 \mathrm{~Hz}$
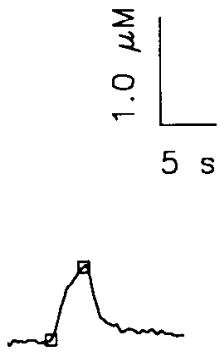

$40 \mathrm{~Hz}$

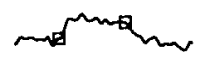

$20 \mathrm{~Hz}$

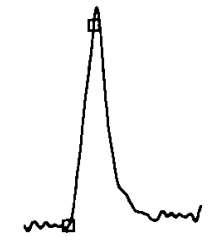

$50 \mathrm{~Hz}$

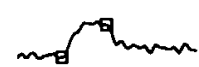

$30 \mathrm{~Hz}$

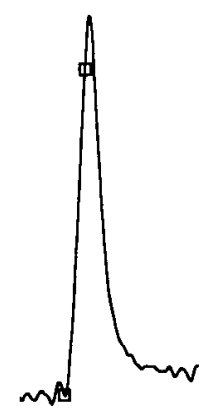

$60 \mathrm{~Hz}$

Figure 4. Frequency dependence of evoked DA overflow in the NAc. Individual overflow curves for $10-60 \mathrm{~Hz}$ are shown. Open boxes denote initiation and termination of the stimulus.

cending norepinephrine fibers in the dorsal tegmental bundle did not elicit a response (Fig. 1).

Dynamics and frequency dependence of stimulated DA overflow. Figures 3-6 show individual overflow curves measured in the $\mathrm{CP}, \mathrm{NAc}, \mathrm{BAN}$, and $\mathrm{MPFC}$, respectively, evoked by stimulation frequencies between 10 and $60 \mathrm{~Hz}$. The dynamics, the range of extracellular concentrations elicited, and the frequency dependence of DA synaptic overflow were markedly different in the four regions. (Note that time scales are identical for all regions; concentration scales are identical for the $\mathrm{CP}, \mathrm{NAc}$, and BAN but are expanded by a factor of 2 for the MPFC.) In the $\mathrm{CP}$ and NAc, evoked overflow was quite similar. There was a dramatic increase in extracellular DA with increasing frequency. In addition, steady-state concentrations of extracellular DA were obtaincd during the stimulation at lower frequencies $(10-30 \mathrm{~Hz})$ whereas the concentration of DA continued to increase throughout the duration of the stimulation at higher frequencies (40$60 \mathrm{~Hz}$ ).

By comparison, the evoked overflow of DA was very different in the BAN and MPFC. A striking difference was observed in the shape of the evoked overflow curves. Overflow curves rose and fell much more slowly in the BAN and MPFC than in the $C P$ and NAc. In addition, the concentration of extracellular DA did not reach steady state in the BAN and MPFC but continued to increase during the course of the stimulation even at the lowest frequencies examined. The frequency dependence of evoked overflow was also differentially expressed in the BAN and MPFC compared to the CP and NAc. Although DA concentrations tended to increase with frequency in the BAN and MPFC, the increase was far less dramatic. In fact, evoked concentrations at high frequencies were relatively frequency independent.

The average responses for the maximum concentration of DA evoked at frequencies between 10 and $60 \mathrm{~Hz}$ are shown in Figure 7 for the four regions. The marked differences in the frequency dependence of overflow as suggested by the individual overflow curves (Figs. 3-6) were clearly seen. In particular, the dramatic increases of extracellular DA in the CP and NAc at the higher frequencies and the relative frequency independence of evoked

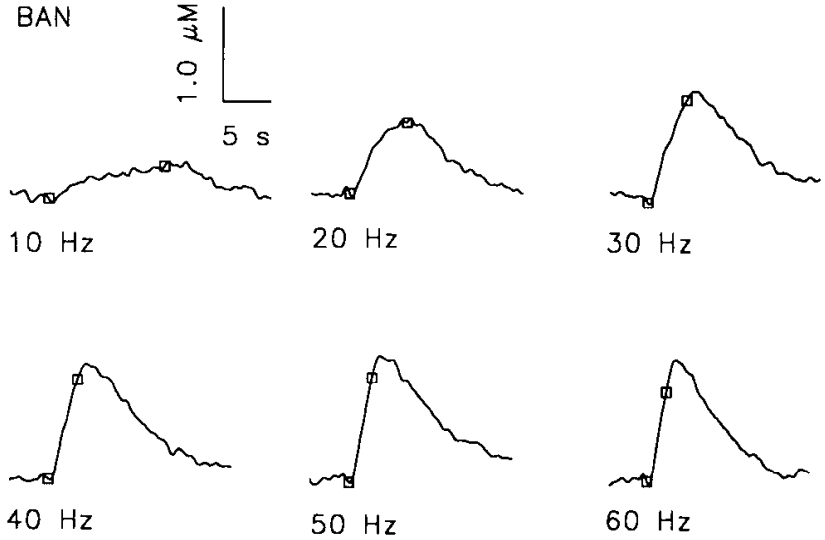

Figure 5. Frequency dependence of evoked DA overflow in the BAN. Individual overflow curves for $10-60 \mathrm{~Hz}$ are shown. Open boxes denote initiation and termination of the stimulus.

DA in the MPFC and BAN were very apparent. A surprising observation was that the magnitude of the absolute concentration elicited by 10 and $20 \mathrm{~Hz}$, that is, frequencies near the physiological range (Chiodo, 1988; Bunney et al., 1991), were not significantly different between the regions

Comparison of DA uptake. We attribute the qualitative differences observed in the overflow curves recorded in the four regions to differences in DA uptake and release. In the next two sections, we provide quantitative information concerning rates for uptake and release in order to compare directly these parameters in the four regions.

The extracellular clearance of evoked DA after the stimulation is due to cellular uptake of DA (Ewing and Wightman, 1984; Wightman and Zimmerman, 1990), and the rate of this process can be determined from these curves. Examination of the individual clearance curves overlaid in Figure 8 clearly showed that uptake is much slower in the MPFC and BAN than in the $\mathrm{CP}$ and NAc. This was also demonstrated by comparing the average rate constants for DA uptake summarized in Table 1 for each region. Uptake was similar in the MPFC and BAN and significantly less $(P<0.001)$ than that in the CP and NAc. The significantly $(P<0.003)$ greater rate of uptake in the CP compared to the NAc has been previously reported, and is due to differences in $V_{\max }$ (May and Wightman, 1989a; Marshall et al., 1990).

Comparison of $D A$ release. Initial rates of DA appearance in the extracellular space following stimulation were much greater in the CP and NAc than in the MPFC and BAN (Figs. 3-6). This suggests that DA release rates must be higher in these regions of the striatum. However, due to the contribution of uptake, only by modeling can a true release parameter be gleaned from the overflow curves. The release parameter we obtain is $[\mathrm{DA}]_{f}$, the concentration of DA per stimulus pulse. In previous work, we have shown that $[\mathrm{DA}]_{n}$, is essentially constant with stimulus duration and frequency above $10 \mathrm{~Hz}$ in the $\mathrm{CP}$ and NAc; furthermore, $[\mathrm{DA}]_{p}$ is unchanged by uptake inhibitors in these regions, suggesting that it is a fundamental index of release (Wightman et al., 1988; May and Wightman, 1989a; Kawagoe et al., 1992). Table 2 shows the relationship between $[\mathrm{DA}]_{p}$ and frequency in MPFC, BAN, NAc, and CP. There was no significant effect of frequency on $[\mathrm{DA}]_{p}$ in any region, although a slight trend was observed for an increasing [DA], with decreasing frequency in the CP but not in the other three regions. Therefore, 

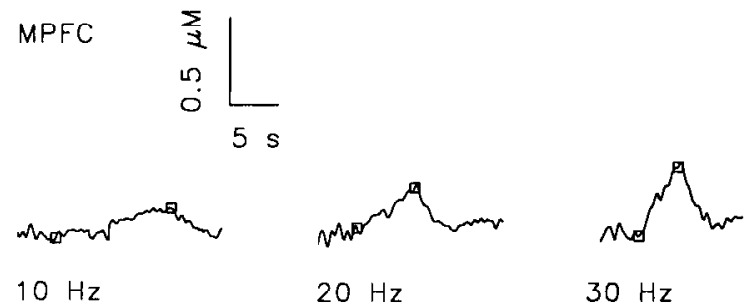

$30 \mathrm{~Hz}$

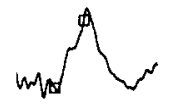

$40 \mathrm{~Hz}$

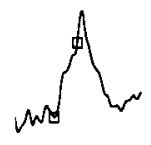

$50 \mathrm{~Hz}$

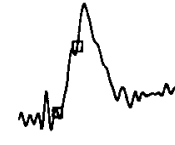

$60 \mathrm{~Hz}$

Figure 6. Frequency dependence of evoked DA overflow in the MPFC. Individual overflow curves for $10-60 \mathrm{~Hz}$ are shown. Open boxes denote initiation and termination of the stimulus.

$[\mathrm{DA}]_{p}$ was averaged over these frequencies in each animal and the mean values are summarized in Table 1 for each region. As expected, $[\mathrm{DA}]_{p}$ was significantly greater $(P<0.001)$ in the CP and NAc than in the MPFC and BAN. No differences were observed between the $\mathrm{CP}$ and NAc, in accord with our previous report (May and Wightman, 1989a), or between the MPFC and BAN.

Kinetic analysis of evoked DA overflow. An important test of the model is to use a single set of kinetic parameters to describe the data obtained in one region at all frequencies. As seen in Figures 7,9, and 10, the rate constants described the measured responses quite well. In Figure 7 , maximum concentrations evoked at each frequency were compared to the simulated responses shown by the solid line. The averaged rate constants summarized in Table 1 were used to predict concentrations for all frequencies in each brain region. In Figure 9, individual

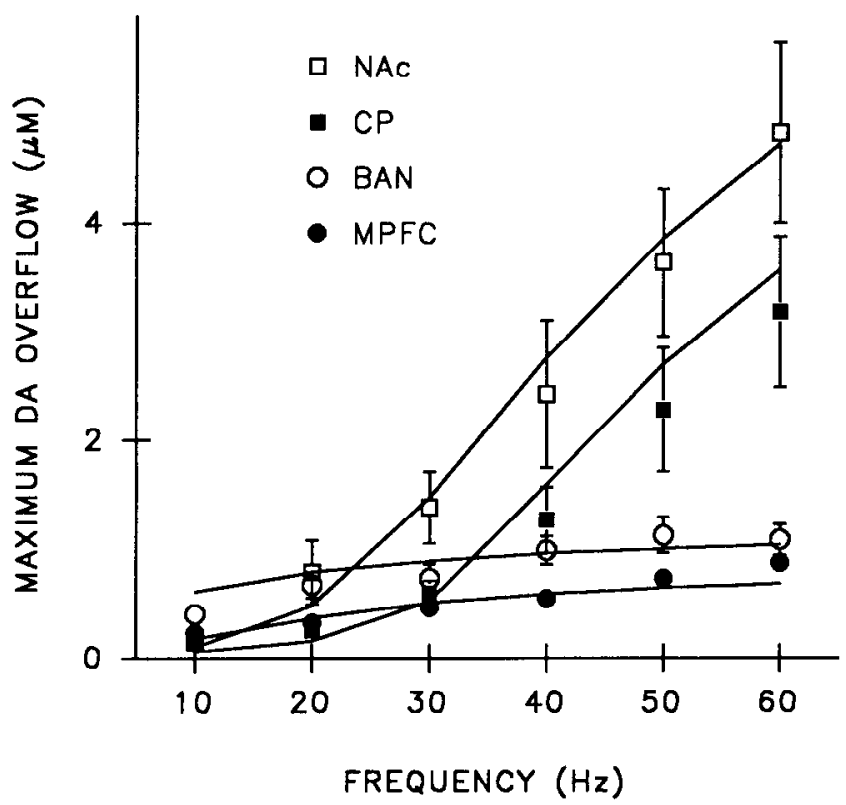

Figure 7. Frequency dependence of maximum overflow. Each point is the mean \pm SEM from at least two rats and each region was examined in five different animals. Solid lines represent averaged maximum concentrations calculated using the reported rate constants in Table 1.

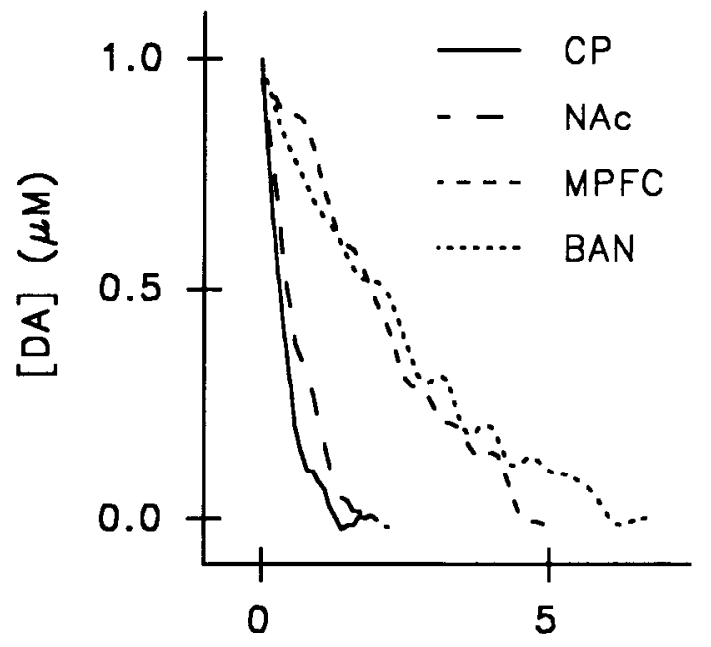

TIME (s)

Figure 8. Extracellular clearance of evoked DA. Individual clearance curves were derived from evoked overflow curves after the stimulus had ceased and were overlaid at a concentration of approximately $1 \mu \mathrm{M}$.

overflow curves and the simulated response for a single frequency were superimposed. Release and uptake parameters used for each simulation were derived from all frequencies in the individual rat. In Figure 10, the simulated response in the BAN for all frequencies is shown. Kinetic values used in the simulations were determined from data in Figure 5 and held constant at each frequency. The frequency independence of evoked overflow at frequencies of $\geq 30 \mathrm{~Hz}$ observed in the measured response is also clearly evident in simulated data. The diffusional component present in overflow curves, as evidenced by the continued rise in extracellular DA concentration after the stimulus, is fully accounted for by diffusion of DA through the Nafion electrode coating.

\section{Discussion}

The objective of this study was to compare the regulation of extracellular DA levels in four projection fields of mesotelencephalic DA neurons of the rat brain. Specifically, we analyzed changes in extracellular DA concentration as a result of transient electrical stimulation of ascending DA fibers in order to assess the characteristics of DA release and uptake. We show marked differences in the dynamics, frequency dependence, and clearance rates of the evoked synaptic overflow of DA in the MPFC, BAN, CP, and NAc. These differences are a direct consequence of different rates of DA release and uptake in the four regions. These results are significant in that the characteristics of mesotelencephalic DA neurons are further differentiated. The factors of release and uptake along with diffusion directly determine functional, that is, extracellular, levels of DA in the brain in time and space and are thus fundamental to determining the nature of DA neural transmission.

Meaningful evaluation of extracellular neurotransmitter dynamics requires the capability to make spatially and temporally resolved measurements. The temporal resolution of FSCV and the spatial resolution afforded by the carbon-fiber microelectrodes (May and Wightman, 1989b) have previously allowed this goal to be achieved in the CP and NAc (Wightman et al., 


\begin{tabular}{|c|c|c|c|c|c|}
\hline \multirow[b]{2}{*}{ Region } & \multirow{2}{*}{$\begin{array}{l}\text { Content }^{a} \\
\text { (ng mg } \\
\text { protein }\end{array}$} & \multicolumn{2}{|c|}{ Absolute } & \multicolumn{2}{|c|}{ Relative $^{h}$} \\
\hline & & $\begin{array}{l}{[\mathrm{DA}]_{,},} \\
\text {(nM) }\end{array}$ & $\begin{array}{l}k \\
\left(\sec { }^{\prime}\right)\end{array}$ & {$[\mathrm{DA}]_{p}$} & $k$ \\
\hline MPFC & 1 & $\begin{array}{l}10.7 \\
\pm 0.8\end{array}$ & $\begin{array}{c}0.53 \\
\pm 0.06\end{array}$ & 10 & 0.5 \\
\hline BAN & 15 & $\begin{array}{l}13.8 \\
\pm 1.7\end{array}$ & $\begin{array}{c}0.32 \\
\pm 0.06\end{array}$ & 1 & 0.02 \\
\hline NAc & 90 & $\begin{array}{l}67.5 \\
\pm 13.4\end{array}$ & $\begin{array}{l}13.9 \\
\pm 2.4\end{array}$ & 1 & 0.2 \\
\hline $\mathrm{CP}$ & 90 & $\begin{array}{l}89.3 \\
\pm 12.3\end{array}$ & $\begin{array}{l}22.7 \\
\pm 3.0\end{array}$ & 1 & 0.3 \\
\hline
\end{tabular}

"Representative tissue contents of DA expressed as ng mg protein ${ }^{-1}$ (Palkovits et al., 1979; Kilts and Anderson, 1987; Marshall et al., 1990). For the MPFC and striatum, DA tissue contents correlate directly with DA terminal density (see Doucet et al., 1986; Descarries et al., 1987).

"Data are absolute values for [DA], and $k$ divided by tissue content. Units are nM $\mathrm{mg}$ protein $\mathrm{ng}^{-1}$ for relative $[\mathrm{D} \Lambda]_{i}$, and $\mathrm{sec}^{-1} \mathrm{ng} 1 \mathrm{mg}$ protein for relative $k$.

' Data are the mean $\pm \operatorname{SEM}(n=5$ animals for each region).

1988; May and Wightman, 1989a; Wightman and Zimmerman, 1990; Kawagoe et al., 1992). Prior work has shown that the use of a broad range of stimulation frequencies enables differences in release and uptake to be revealed (May and Wightman, 1989a). The results reported herein demonstrate the utility of this technique to study the regulation of extracellular DA levels in DA terminal fields other than the striatum.

Differences in the extracellular clearance of evoked DA in the four regions are clearly evident when the descending portions of the overflow curves are compared (Fig. 8). In the striatum, uptake precedes metabolism (Near et al., 1988), a slow process compared to the time scale of evoked DA overflow (Michael et al, 1985). Similarly, diffusion occurs at a much slower rate (Ewing and Wightman, 1984). Therefore, we attribute extracellular clearance as a real-time observation of uptake. Quantitative analysis of these curves shows that uptake rates are significantly less in the MPFC and BAN than the CP and NAc.

$A$ very different picture emerges when uptake is normalized to tissue DA content in order to account for differences in DA terminal density (Table 1). The normalized rate constants for

\section{Table 2. Relationship between the frequency of stimulation and} $[\mathrm{DA}]_{p}^{a}$

Frequency $(\mathrm{Hz})$

\begin{tabular}{lcccll} 
Region & 20 & 30 & 40 & 50 & 60 \\
\hline MPFC & 9.9 & 9.3 & 9.6 & 11.5 & 12.8 \\
& $\pm 1.2^{\prime \prime}$ & \pm 1.2 & \pm 0.7 & \pm 0.9 & \pm 0.9 \\
BAN & 12.0 & 12.4 & 14.4 & 15.6 & 14.6 \\
& \pm 1.8 & \pm 1.5 & \pm 1.9 & \pm 2.0 & \pm 1.9 \\
NAc & 65.0 & 63.4 & 65.6 & 66.4 & 73.0 \\
& \pm 13.5 & \pm 8.9 & \pm 9.9 & \pm 7.5 & \pm 9.3 \\
CP & 102.4 & 93.2 & 84.2 & 83.2 & 84.4 \\
& \pm 16.1 & \pm 13.1 & \pm 11.6 & \pm 10.9 & \pm 11.0
\end{tabular}

"[DA], is given as nM. There was no significant effect of frequency on [DA] $(F=0.106, \mathrm{df}=4,75)$. There was a significant $(P<0.001)$ effect of region on $[D A]_{n}(F=89.9, \mathrm{df}=3,75)$.

"Data are the mean $\pm \operatorname{SEM}(n=5$ animals for each region).
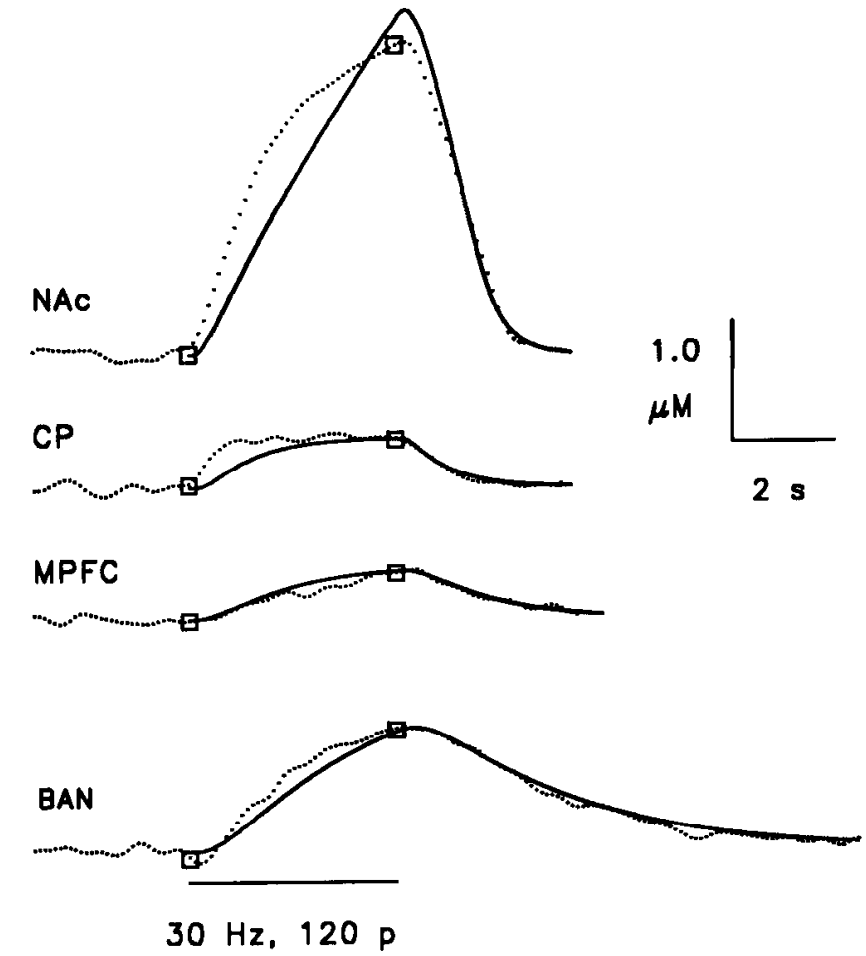

Figure 9. Comparison of the overflow curves obtained at $30 \mathrm{~Hz}$ in the four different regions. Individual overflow curves from $4 \mathrm{sec}$ stimulations are shown. Data points collected every $100 \mathrm{msec}$ are represented by dots. Solid lines are the calculated responses using kinetic parameters determined from stimulated overflow at frequencies from 20 to $60 \mathrm{~Hz}$ in the same animal (see Materials and Methods for details) and convoluted with diffusion through Nafion. Open boxes denote initiation and termination of the stimulus.

uptake are similar in the MPFC, NAc, and CP, but it is at least 10 times less in the BAN. Thus, uptake in the BAN is not related to terminal density in the same manner as the other regions and must be distinct. The uptake of DA in the CP and NAc is

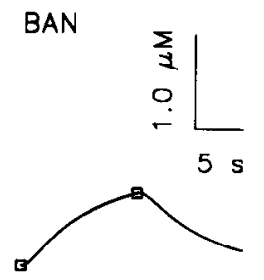

$10 \cdot \mathrm{Hz}$

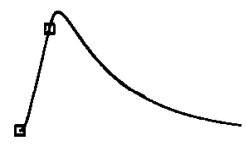

$40 \mathrm{~Hz}$

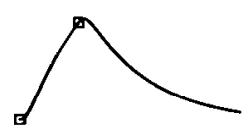

$20 \mathrm{~Hz}$

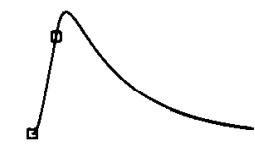

$50 \mathrm{~Hz}$

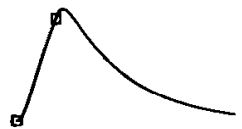

$30 \mathrm{~Hz}$

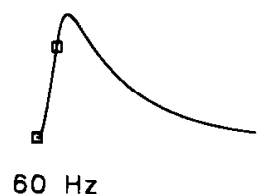

Figure 10. Simulations of evoked DA overflow in the BAN. Kinetic constants for relcase $\left([\mathrm{DA}]_{p}=14.6 \mathrm{nM}\right)$ and uptake $\left(k=0.15 \mathrm{sec}^{-1}\right)$ were determined from the experimental data shown in Figure 5. All kinetic constants were identical for simulations at each frequency. Simulations were convoluted using a thin layer distance of $400 \mathrm{~nm}$ and a diffusion coefficient for DA in Nafion of $1 \times 10^{-9} \mathrm{~cm}^{2} \mathrm{sec}^{-1}$. The number of stimulus pulses was 120 . Open boxes denote initiation and termination of the stimulus. Concentration and time scales are identical with Figure 5. 
characterized as high affinity with a $K_{\mathrm{m}}$ of around $0.16 \mu \mathrm{M}$ (Near et al., 1988; Marshall et al., 1990). Whether the slow rates of DA uptake in the BAN represent low-affinity uptake similar to that found in the neuroendocrine tuberoinfundibular DA system (Demarest and Moore, 1979; Annunziato et al., 1980) and/or a reduced number of uptake sites per terminal is not at present known. The gene for the rat DA transporter has recently been cloned (Kilty et al., 1991; Shimada et al., 1991) and it is tempting to speculate that the DA transporter present in the BAN may be structural different, possibly as a result of posttranslational modification, from that in the other regions.

The different absolute rates of uptake in the four regions are directly responsible for the observed differences in the frequency dependence of maximum overflow. We have previously shown in the $\mathrm{CP}$ and $\mathrm{NAc}$ that the marked increases in concentration with frequency are not due to increasing rates of release but rather are duc to the ratc of uptake (Wightman and Zimmcrman, 1990). As the stimulation frequency increases, less time is available between stimulus pulses for uptake to exert its effect and, thus, the maximal concentration of DA in the CP and NAC progressively increases. Note, however, that maximum overflow in the MPFC and BAN is much less dependent on frequency. In fact, maximum overflow at frequencies of $>30 \mathrm{~Hz}$ is almost independent of frequency in the MPFC and BAN. The combination of inefficient uptake and a reduced time at high frequencies renders uptake negligible. Thus, maximum overflow at high frequencies in these regions is primarily dependent on the number of pulses in the stimulus train, which was constant at all frequencies in these experiments.

To obtain a quantitative estimate of release rates, we have employed a kinetic model that permits resolution of the observed synaptic overflow during stimulation into release and uptake components. Comparison between regions is appropriate because the stimulation amplitude was maximal for all regions. Parameters that describe DA release are found to be similar in the MPFC and BAN but approximately eight times less than that in the CP and NAc. However, when normalized to DA tissue content, relative release is similar in the BAN, NAc, and $\mathrm{CP}$, but 10 times greater in the MPFC. This finding is in agreement with the greater fractional overflow of evoked tritiated DA in vitro from slices of the MPFC compared to the striatum (Hoffmann et al., 1988) and may contribute to the relatively high concentrations of extracellular DA in the MPFC measured by dialysis (Sharp et al., 1986). Since mesoprefrontal DA neurons fire at a faster mean rate compared to mesostriatal DA neurons (Chiodo, 1988), they may have developed a more proficient secretory machinery to sustain high release. Differences in the autoregulation of release are unlikely to be responsible since all of the DA systems examined exhibit autoreceptors governing release (Talmaciu et al., 1986; Altar et al., 1987; Kilts et al., 1987; Moghaddam and Bunney, 1990; Bean and Roth, 1991; Bull et al., 1991). However, differences in feedback loops regulating mesotelencephalic DA systems might contribute to the differences in release rates (Thierry et al., 1979).

Thus, comparison of the normalized kinetic parameters for DA release and uptake reveals that the regulation of extracellular DA levels is, indeed, unique in the MPFC, BAN, and striatum. These differences explain the striking observation that maximum evoked overflow was similar in the MPFC, BAN, NAc, and $C P$ at 10 and $20 \mathrm{~Hz}$, frequencies close to the physiological range (Chiodo, 1988; Bunney et al., 1991), despite the great disparity in the densities of the DA terminal fields. Based on
DA tissue contents alone (Table 1), extracellular DA concentrations should be 15- and 90-fold less in the BAN and MPFC, respectively, than in the CP or NAc. We contend that the unusually high DA concentrations in the MPFC are the result of a distinctly high rate of DA release and that the unusually high concentrations in the BAN are the result of a distinctly low rate of DA uptake.

Another useful way to characterize the dissimilar regulation of extracellular DA levels in the four regions is to use the ratio of release to uptake rates as an index. This ratio is relatively small in the CP and NAc, and these areas can be considered "uptake dominated." A consequence of this regulation is the observation of steady-state concentrations of extracellular DA in the CP and NAc during stimulation with low frequencies. Steady state is obtained because release and uptake rates are balanced (Wightman et al., 1988). The capacity for a high rate of overflow only becomes manifested at higher frequencies when the time for uptake decreases. In contrast, the ratio of release to uptake in the MPFC and BAN is 5-10 times larger than the striatal regions, so these regions can be considered "release dominated," even though different mechanisms are involved. As a consequence of this type of regulation, steady-state concentrations of extracellular DA are not reached, even at low frequencies, because release rates exceed that of uptake rates.

The absolute uptake rates can, in particular, exert profound effects on the spatial dynamics of DA because they dictate its extracellular lifetime. During stimulation all of the terminals are forced to fire simultaneously, causing a uniform concentration increase of DA in the extracellular fluid. However, when a single terminal is activated, a concentration gradient will exist, and diffusion can occur (Kawagoe et al., 1992). The diffusional dispersion of DA that has escaped the synapse into the extracellular space will occur until it is taken up into cells. The halflife for uptake is approximately $2.0 \mathrm{sec}$ in the MPFC and BAN, in contrast to $<0.06 \mathrm{sec}$ in the CP and NAc. Thus, in one halflife, extracellular DA could diffuse a sixfold greater distance from the synapse in the more sparsely innervated areas.' The overall regulation of extracellular DA levels in the MPFC and $\mathrm{BAN}$ is therefore well suited for generating concentration gradients for DA over a widespread area surrounding release sites, which is in sharp contrast to that in the striatum. While uptakedominated regulation in the $C P$ and NAc agrees with the expectations for a classical, synaptic neurotransmitter, the releasedominated regulation in the MPFC and BAN could not only support synaptic communication, but also communication with other, quite distant sites.

To date, the concept of nonsynaptic neural transmission in the CNS (Fuxe and Agnati, 1991; Vizi and Labos, 1991) has been primarily based on anatomical evidence, for example, documenting mismatches between neurotransmitter and receptor localizations (Herkenham, 1987) and the absence of synaptic junctional complexes (Séguela et al., 1989, 1990) for certain neuronal systems. However, the hypothesis is quite controversial, especially for classical neurotransmitters such as monoamines (Bloom, 1991), and is difficult to assess solely on the basis of anatomical features. Examination of extracellular neurotransmitter dynamics adds another dimension to the anatom-

\footnotetext{
'The approximate diffusion distance is given by $\left(D t_{1: 2}\right)^{1 / 2}$, where $D$ is the diffusion coefficient of DA and $t_{1}$, is the extracellular half-life of evoked DA. For $D=6 \times$ $10^{\circ} \mathrm{cm}^{2} \mathrm{sec}^{-1}$, its value in saline at $25^{\circ} \mathrm{C}$, diffusion distances would be $<6 \mu \mathrm{m}$ in the CP and NAc but $30-40 \mu \mathrm{m}$ in the MPFC and BAN.
} 
ical studies. For example, anatomical studies of the monoaminergic innervation of the MPFC (Séguela et al., 1988, 1989, 1990) have shown that dopaminergic terminals exhibit true synaptic contacts with a similar synaptic architecture to that in the striatum (Bouyer et al., 1984; Freund et al., 1984; Séguela et al., 1988), suggesting chemical transmission is synaptic (Descarries et al., 1991). Yet, the regulation of extracellular DA in the MPFC is strikingly different from that in the striatum.

Nonsynaptic dopaminergic transmission certainly occurs in the tuberoinfundibular DA system. These DA neurons meet the anatomical criteria for nonsynaptic neural transmission since they do not make synaptic contacts with their target, the anterior pituitary, a site distant from their terminals (Ben-Jonathan, 1985). In addition, regulation of extracellular DA levels is consistent with what we predict to support nonsynaptic neural transmission: tuberoinfundibular DA neurons have low uptake rates (Demarest and Moore, 1979; Annunziato et al., 1980). Thus, it may be that chemical communication by DA in the MPFC and BAN has characteristics intermediate to that in the median eminence and striatum.

Summary and conclusions. DA terminal fields exhibit distinct mechanisms for governing extracellular DA levels. In general, the regulation can be considered "uptake dominated" in the $\mathrm{CP}$ and NAc and "release dominated" in the MPFC and BAN. This classification may be useful for predicting the effects of drugs that alter DA release and uptake on extracellular DA concentrations in the different regions. In addition, we have shown that "long-range" diffusion of DA is more likely in some brain regions. The consequence could be of profound functional significance: compared to the CP and NAc, extracellular DA has a greater possibility of extrasynaptic sites of action in the MPFC and BAN. This study of mesotelencephalic DA neurons provides a unique example of the differences in modes of chemical neural transmission that can occur as a result of differences in mechanisms that regulate extracellular levels.

\section{References}

Altar CA, Boyar WC, Oei E, Wood PL (1987) Dopamine autoreceptors modulate the in vivo release of dopamine in the frontal, cingulate and entorhinal cortices. J Pharmacol Exp Ther 242:115-120.

Anden NE, Fuxe K, Hamberger B, Hökfelt T (1966) A quantitative study of the nigro-ncostriatal dopamine neuron system in the rat. Acta Physiol Scand 67:306-312.

Annunziato L, Leblanc P, Kordan C, Weiner RI (1980) Differences in the kinetics of dopamine uptake in synaptosome preparations of the median eminence relative to other dopaminergically innervated brain regions. Neuroendocrinology 31:316-320.

Bannon MJ, Roth RH (1983) Pharmacology of mesocortical dopamine neurons. Pharmacol Rev 35:53-68.

Baur JE, Kristensen EW, May LJ, Wiedemann DJ, Wightman RM (1988) Fast-scan voltammetry of biogenic amines. Anal Chem 60: $1268-1272$.

Bean AJ, Roth RH (1991) Extracellular dopamine and neurotensin in rat prefrontal cortex in vivo: effects of median forebrain bundle stimulation frequency, stimulation pattern, and dopamine autoreceptors. J Neurosci 11:2694-2702.

Ben-Jonathan N (1985) Dopamine: a prolactin inhibiting hormone. Endocr Rev 6:564-589.

Björklund A, Lindvall O (1984) Dopamine-containing systems in the CNS. In: Handbook of chemical neuroanatomy (Björklund A, Hökfelt T, eds), pp 55-122. New York: Elsevier.

Bloom FE (1991) An integrative view of information handling in the CNS. In: Volume transmission in the brain: novel mechanisms for neural transmission (Fuxe K, Agnati LF, eds), pp 11-23. New York: Raven.

Bouyer JJ, Joh TH, Pickel VM (1984) Ultrastructural localization of tyrosine hydroxylase in rat nucleus accumbens. I Comp Neurol 227: 92-103.

Bull DR, Bakhtiar R, Sheehan MJ (1991) Characterization of dopamine autoreceptors in the amygdala: a fast cyclic voltammetric study in vitro. Neurosci Lett 134:41-44.

Bunney BS, Chiodo LA, Grace AA (1991) Midbrain dopamine system electrophysiological functioning: a review and new hypothesis. Synapse 9:79-94.

Carlsson M, Carlsson A (1990) Interactions between glutamatergic and monoaminergic systems within the basal ganglia-implications for schizophrenia and Parkinson's disease. Trends Neurosci 13:272276

Chiodo LA (1988) Dopamine-containing neurons in the mammalian central nervous system: electrophysiology and pharmacology. Neurosci Biobehav Rev 12:49-91.

Demarest KT, Moore KE (1979) Lack of a high affinity transport system for dopamine in the median eminence and posterior pituitary. Brain Res 171:545-551.

Descarries L, Lemay B, Doucet G, Berger B (1987) Regional and laminar density of the dopamine innervation in adult rat cerebral cortex. Neuroscience 21:807-824.

Descarries L, Séguela P, Watkins KC (1991) Nonjunctional relationships of monoamine axon terminals in the cerebral cortex of adult rat. In: Volume transmission in the brain: novel mechanisms for neural transmission (Fuxe K, Agnati LF, eds), pp 53-62. New York: Raven.

Doucet G, Descarries L, Garcia S (1986) Quantification of the dopamine innervation in adult rat neostriatum. Neuroscience 19:427445.

Ewing AG, Wightman RM (1984) Monitoring the stimulated release of dopamine with in vivo voltammetry. II: Clearance of released dopamine from extracellular fluid. J Neurochem 43:570-577.

Ewing AG, Bigelow JC, Wightman RM (1983) Direct in vivo monitoring of dopamine released from two striatal compartments in the rat. Science 221:169-171.

Freund TF, Powell JF, Smith AD (1984) Tyrosine hydroxylase-immunoreactive boutons in synaptic contact with identified striatonigral neurons, with particular reference to dendritic spines. Neuroscience 13:1189-1215.

Fuxe K, Agnati LF (1991) Two principal modes of electrochemical communication in the brain: volume versus wiring transmission. In: Volume transmission in the brain: novel mechanisms for neural transmission (Fuxe K, Agnati LF, eds), pp 1-9. New York: Raven.

Garris PA, Collins LB, Jones SR, Wightman RM (1993) Evoked extracellular dopamine in vivo in the medial prefrontal cortex. J Neurochem $61: 637-647$.

Gerfen CR, Herkenham R, Thibault J (1987) The neostriatal mosaic: II. Patch- and matrix-directed mesostriatal dopaminergic and nondopaminergic systems. J Neurosci 7:3915-3934

Glowinski J, Tassin JP, Thierry AM (1984) The mesocortico-prefrontal dopaminergic neurons. Trends Neurosci 7:414-418.

Goldman-Rakic PS (1984) The frontal lobes: uncharted provinces of the brain. Trends Neurosci 11:425-429.

Herkenham M (1987) Mismatches between neurotransmitter and receptor localizations in brain: observations and implications. Neuroscience 23:1-38.

Hoffmann IS, Talmaciu RK, Ferro CP, Cubeddu LX (1988) Sustained high release at rapid stimulation rates and reduced functional autoreceptors characterize prefrontal cortex dopamine terminales. J Pharmacol Exp Ther 245:761-772.

Justice JR Jr, Nicolaysen LC, Michael AC (1988) Modelling the dopaminergic nerve terminal. J Neurosci Methods 22:239-252.

Kawagoe KT, Garris PA, Wiedemann DJ, Wightman RM (1992) Regulation of transient dopamine concentration gradients in the microenvironment surrounding nerve terminals in the rat striatum. Neuroscience 51:55-64.

Kilts CD, Anderson CM (1987) Mesoamygdaloid dopamine neurons: differential rates of dopamine turnover in discrete amygdaloid nuclei of the rat brain. Brain Res 416:402-408.

Kilts CD, Anderson CM, Ely TD, Nishita JK (1987) Absence of synthesis-modulating nerve terminal autoreceptors on mesoamygdaloid and other mesolimbic dopamine neuronal populations. J Neurosci 7:3961-3975.

Kilts CD, Anderson CM, Ely TD, Mailman RB (1988) The biochem- 
istry and pharmacology of mesoamygdaloid dopamine neurons. Ann NY Acad Sci 537:173-187.

Kilty JE, Lorange D, Amara SG (1991) Cloning and expression of a cocaine-sensitive rat dopamine transporter. Science 254:578-579.

Koob GF, Stinus L, Le Moal M, Bloom FE (1989) Opponent process theory of motivation: neurobiological evidence from studies of opiate dependence. Neurosci Biobehav Rev 13:135-140.

Kuhr WG, Ewing AG, Caudill WL, Wightman RM (1984) Monitoring the stimulated release of dopamine with in vivo voltammetry. I: Characterization of the response observed in the caudate nucleus of the rat. J Neurochem 43:560-569.

Kuhr WG, Bigelow JC, Wightman RM (1986) In vivo comparison of the regulation of releasable dopamine in the caudate nucleus and the nucleus accumbens of the rat brain. J Neurosci 6:974-982.

Le Moal M, Simon H (1991) Mesocorticolimbic dopaminergic network: functional and regulatory roles. Physiol Rev 71:155-234.

Marsden CA, Joseph MH, Kruk ZL, Maidment NT, O'Neill RD, Schenk JO, Stamford JA (1988) In vivo voltammetry-present electrodes and methods. Neuroscience 25:389-400.

Marshall JF, O'Dell SJ, Navarrete R, Rosenstein AJ (1990) Dopamine high-affinity transport site topography in rat brain: major differences between dorsal and ventral striatum. Neuroscience 37:11-21.

May LJ, Wightman RM (1989a) Effects of D-2 antagonists on frequency-dependent stimulated dopamine overflow in nucleus accumbens and caudate-putamen. J Neurochem 53:898-906.

May LJ, Wightman RM (1989b) Heterogeneity of stimulated dopamine overflow within the rat striatum as observed with in vivo voltammetry. Brain Res 487:311-320.

Michael AC, Justice JB Jr, Neill DB (1985) In vivo voltammetric determination of the kinetics of dopamine metabolism in the rat. Neurosci Lett 56:365-369.

Michael AC, Ikeda M, Justice JB Jr (1987) Dynamics of the recovery of releasable dopamine following electrical stimulation of the medial forebrain bundle. Neurosci Lett 76:81-86.

Millar J, Stamford JA, Kruk ZL, Wightman RM (1985) Electrochemical, pharmacological and electrophysiological evidence of rapid dopamine release and removal in the rat caudate nucleus following electrical stimulation of the medial forebrain bundle. Eur J Pharmacol 109:341-348.

Moghaddam B, Bunney BS (1990) Acute effects of typical and atypical antipsychotic drugs on the release of dopamine from prefrontal cortex, nucleus accumbens and striatum of the rat: an in vivo microdialysis study. J Neurochem 54:1755-1760.

Near JA, Bigelow JC, Wightman RM (1988) Comparison of uptake of dopamine in rat striatal chopped tissue and synaptosomes. J Pharmacol Exp Ther 245:921-927.

Palkovits M, Zaborszky L, Brownstein MJ, Fekete MIK, Herman JP, Kanyicska B (1979) Distribution of norepinephrine and dopamine in cerebral cortical areas of the rat. Brain Res Bull 4:593-601.

Parsons LH, Justice JB Jr (1992) Extracellular concentration and in vivo recovery of dopamine in the nucleus accumbens using microdialysis. J Neurochem 58:212-218.

Paxinos G, Watson C (1986) The rat brain in stereotaxic coordinates. New York: Academic.

Ross SB (1991) Synaptic concentration of dopamine in the mouse striatum in relationship to the kinetic properties of the dopamine receptors and uptake mechanism. J Neurochem 56:22-29.

Séguela P, Watkins KC, Descarries L (1988) Ultrastructural features of dopamine axon terminals in the anteromedial and suprarhinal cortex of adult rat. Brain Res 442:11-22.
Séguela P, Watkins KC, Descarries L (1989) Ultrastructural relationships of serotonin axon terminals in cerebral cortex of adult rat. J Comp Neurol 289:129-142.

Sćgucla P, Watkins KC, Descarrics L (1990) Noradrenaline axon terminals in adult rat neocortex: an immunocytochemical analysis in serial thin sections. Neuroscience 35:249-264.

Sharp T, Zetterstrom T, Ungerstedt $U$ (1986) An in vivo study of dopamine release and metabolism in rat brain regions using intracerebral dialysis. J Neurochem 47:113-122.

Shimada S, Kitayama S, Lin C-L, Patel A, Nanthakumar E, Gregor P Kuhar M, Uhl G (1991) Cloning and expression of a cocaine-sensitive dopamine transporter complementary DNA. Science 254:576578

Sokal RR, Rohlf FJ (1981) Biometry. San Francisco: Freeman.

Stamford JA, Kruk ZL, Millar J (1988) Stimulated limbic and striatal dopamine release measured by fast cyclic voltammetry: anatomical, electrochemical and pharmacological characterization. Brain Res 454 $282-288$.

Suaud-Chagny MF, Ponec J, Gonon F (1991) Presynaptic autoinhibition of the electrically evoked dopamine release studied in the rat olfactory tubercle by in vivo electrochemistry. Neuroscience 45:641652.

Talmaciu RK, Hoffmann IS, Cubeddu LX (1986) Dopamine autoreceptors modulate dopamine release from the prefrontal cortex. $\mathrm{J}$ Neurochem 47:865-870.

Thierry AM, Deniau JM, Feger J (1979) Effects of stimulation of the frontal cortex on identified output VMT cells in the rat. Neurosci Lett 15:103-107

Vanderwolf CH, Kelly ME, Kraemer P, Streather A (1988) Are emotion and motivation localized in the limbic system and nucleus accumbens? Behav Brain Res 27:45-58.

Vizi ES, Labos E (1991) Non-synaptic interactions at presynaptic level. Prog Neurobiol 37:145-163.

Wiedemann DJ, Kawagoe KT, Kennedy RT, Ciolkowski EL, Wightman RM (1991) Strategies for low detection limit measurements with cyclic voltammetry. Anal Chem 63:2965-2970.

Wiedemann DJ, Garris PA, Near JA, Wightman RM (1992) Effect of chronic haloperidol treatment on stimulated synaptic overflow dopamine in the rat striatum. J Pharmacol Exp Ther 261:574-579.

Wightman RM, Zimmerman JB (1990) Control of dopamine extracellular concentration in rat striatum by impulse flow and uptake. Brain Res Rev 15:135-144.

Wightman RM, Brown DS, Kuhr WG, Wilson RL (1987) Molecular specificity of in vivo electrochemical measurements. In: Voltammetry in the neurosciences (Justice JB Jr, ed), pp 103-138. Clifton, NJ: Ilumana.

Wightman RM, Amatore C, Engstrom RC, Hale PD, Kristensen EW, Kuhr WG, May LJ (1988) Real-time characterization of dopamine overflow and uptake in the rat striatum. Neuroscience 25:513-523.

Williams GV, Millar J (1990a) Differential actions of endogenous and iontophoretic dopamine in rat striatum. Eur J Neurosci 2:658-661.

Williams GV, Millar J (1990b) Concentration-dependent actions of stimulated dopamine release on neuronal activity in rat striatum. Neuroscience 39:1-16.

Wise RA, Hoffman DC (1992) Localization of drug reward mechanisms by intracranial injections. Synapse 10:247-263.

Zigmond MJ, Abercrombie ED, Berger TW, Grace AA, Stricker EM (1990) Compensations after lesions of central dopaminergic neurons: some clinical and basic implications. Trends Neurosci 13:290-295. 